\title{
Low energy atmospheric muon neutrinos in MACRO
}

\author{
MACRO Collaboration
}

\begin{abstract}
M. Ambrosio ' , R. Antolini g , G. Auriemma ${ }^{\mathrm{n}, 1}$, D. Bakari ${ }^{\mathrm{b}, \mathrm{q}}$, A. Baldini ${ }^{\mathrm{m}}$, G.C. Barbarino ${ }^{1}$, B.C. Barish ${ }^{\mathrm{d}}$, G. Battistoni ${ }^{\mathrm{f}, 2}$, R. Bellotti ${ }^{\mathrm{a}}$, C. Bemporad ${ }^{\mathrm{m}}$, P. Bernardini ${ }^{\mathrm{j}}$, H. Bilokon ${ }^{\mathrm{f}}$, V. Bisi ${ }^{\mathrm{p}}, \mathrm{C}$. Bloise ${ }^{\mathrm{f}}, \mathrm{C}$. Bower ${ }^{\mathrm{h}}, \mathrm{M}$. Brigida a ${ }^{\text {a }}$ S. Bussino ${ }^{\text {r }}$, F. Cafagna a , M. Calicchio ${ }^{\text {a }}$, D. Campana ${ }^{\text {}, ~ M . ~ C a r b o n i ~}{ }^{\text {f }}$, S. Cecchini ${ }^{\text {b,3 }}$, F. Cei ${ }^{\text {k,m }}$, V. Chiarella ${ }^{\text {f }}$, B.C. Choudhary ${ }^{\mathrm{d}}$, S. Coutu ${ }^{\mathrm{k}, 4}$, G. De Cataldo a , H. Dekhissi ${ }^{\text {b,q }}$, C. De Marzo a ${ }^{a}$ I. De Mitri ${ }^{j}$, J. Derkaoui ${ }^{\text {b,q }}$,
\end{abstract} M. De Vincenzi ${ }^{\text {r }}$, A. Di Credico ${ }^{\text {g }}$, O. Erriquez ${ }^{a}$, C. Favuzzi ${ }^{a}$, C. Forti ${ }^{\text {f }}$, P. Fusco a , G. Giacomelli ${ }^{\text {b }}$, G. Giannini ${ }^{\mathrm{m}, 5}$, N. Giglietto ${ }^{\mathrm{a}}$, M. Giorgini ${ }^{\mathrm{b}}$, M. Grassi ${ }^{\mathrm{m}}$, L. Gray ${ }^{\mathrm{g}}$, A. Grillo ${ }^{\mathrm{g}}$, F. Guarino ${ }^{1}$, C. Gustavino ${ }^{\mathrm{g}}$, A. Habig ${ }^{\mathrm{c}}$, K. Hanson ${ }^{\mathrm{k}}$, R. Heinz ${ }^{\text {h }}$, E. Iarocci ${ }^{\text {f,6 }}$, E. Katsavounidis ${ }^{\text {d }}$, I. Katsavounidis ${ }^{\text {d }}$, E. Kearns ${ }^{\text {c }}$, H. Kim ${ }^{\text {d }}$, S. Kyriazopoulou ${ }^{\text {d }}$, E. Lamanna ${ }^{\text {n,7 }}$, C. Lane ${ }^{\text {e }}$, D.S. Levin ${ }^{k}$, P. Lipari ${ }^{\text {n, }}$ N.P. Longley ${ }^{\mathrm{d}, 8}$, M.J. Longo ${ }^{\mathrm{k}}$, F. Loparco ${ }^{\mathrm{a}}$, F. Maaroufi ${ }^{\mathrm{b}, \mathrm{q}}, \mathrm{G}$, Mancarella ${ }^{\mathrm{j}}$, G. Mandrioli ${ }^{\text {b }}$, A. Margiotta ${ }^{\text {b }}$, A. Marini ${ }^{\text {f }}$, D. Martello ${ }^{j}$, A. Marzari-Chiesa ${ }^{\text {p }}$, M.N. Mazziotta ${ }^{\text {a }}$, D.G. Michael ${ }^{\text {d }}$, S. Mikheyev ${ }^{\text {d,g, }, 9}$, L. Miller ${ }^{\text {h,10 }}$, P. Monacelli i, T. Montaruli a , M. Monteno ${ }^{\mathrm{p}}, \mathrm{S}$. Mufson h ${ }^{\mathrm{h}}$ J. Musser ${ }^{\mathrm{h}}$, D. Nicolò ${ }^{\mathrm{m}, 11}$, R. Nolty ${ }^{\mathrm{d}}$, C. Orth ${ }^{\mathrm{c}}$, G. Osteria ${ }^{\text {l }}$, M. Ouchrif ${ }^{\mathrm{b}, \mathrm{q}}$, O. Palamara ${ }^{\mathrm{g}}$, V. Patera ${ }^{\mathrm{f}, 6}$, L. Patrizii $^{\mathrm{b}}$, R. Pazzi ${ }^{\mathrm{m}}$, C.W. Peck ${ }^{\mathrm{d}}$, L. Perrone ${ }^{\mathrm{j}}$, S. Petrera ${ }^{\mathrm{i}}$, P. Pistilli ${ }^{\mathrm{r}}$, V. Popa ${ }^{\mathrm{b}, 12}$, A. Rainò a , J. Reynoldson ${ }^{\text {g }}$, F. Ronga ${ }^{\text {f }}$, C. Satriano ${ }^{\mathrm{n}, 1}$, L. Satta ${ }^{\mathrm{f}, 6}$, E. Scapparone ${ }^{\mathrm{g}}$, K. Scholberg ${ }^{\text {c }}$, A. Sciubba ${ }^{\mathrm{f}, 6}$, P. Serra ${ }^{\text {b }}$, M. Sioli ${ }^{\text {b }}$, G. Sirri ${ }^{\text {b,g }}$, M. Sitta ${ }^{\text {, }}$, P. Spinelli a , M. Spinetti ${ }^{\mathrm{f}}$, M. Spurio ${ }^{\mathrm{b}, 13}$, R. Steinberg ${ }^{\mathrm{e}}$, J.L. Stone ${ }^{\mathrm{c}}$, L.R. Sulak ${ }^{\mathrm{c}}$, A. Surdo ${ }^{j}$, G. Tarlè k, V. Togo ${ }^{\text {b }}$, M. Vakili ${ }^{\circ}$, E. Vilela ${ }^{b}$, C.W. Walter ${ }^{c, d}$, R. Webb ${ }^{\circ}$

\footnotetext{
a Dipartimento di Fisica dell'Università di Bari and INFN, 70126 Bari, Italy

b Dipartimento di Fisica dell'Università di Bologna and INFN, 40126 Bologna, Italy c Physics Department, Boston University, Boston, MA 02215, USA

${ }^{\mathrm{d}}$ California Institute of Technology, Pasadena, CA 91125, USA

e Department of Physics, Drexel University, Philadelphia, PA 19104, USA

${ }^{\mathrm{f}}$ Laboratori Nazionali di Frascati dell'INFN, 00044 Frascati (Roma), Italy

g Laboratori Nazionali del Gran Sasso dell'INFN, 67010 Assergi (L'Aquila), Italy

${ }^{\mathrm{h}}$ Depts. of Physics and of Astronomy, Indiana University, Bloomington, IN 47405, USA

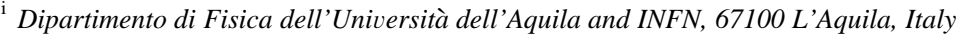




\author{
${ }^{\mathrm{j}}$ Dipartimento di Fisica dell'Università di Lecce and INFN, 73100 Lecce, Italy \\ ${ }^{\mathrm{k}}$ Department of Physics, University of Michigan, Ann Arbor, MI 48109, USA \\ ${ }^{1}$ Dipartimento di Fisica dell'Università di Napoli and INFN, 80125 Napoli, Italy \\ ${ }^{\mathrm{m}}$ Dipartimento di Fisica dell'Università di Pisa and INFN, 56010 Pisa, Italy \\ "Dipartimento di Fisica dell'Università di Roma 'La Sapienza"' and INFN, 00185 Roma, Italy \\ ${ }^{\circ}$ Physics Department, Texas A \& M University, College Station, TX 77843, USA \\ ${ }^{\mathrm{p}}$ Dipartimento di Fisica Sperimentale dell'Università di Torino and INFN, 10125 Torino, Italy \\ ${ }^{\mathrm{q}}$ L.P.T.P., Faculty of Sciences, University Mohamed I, B.P. 524 Oujda, Morocco \\ ${ }^{\mathrm{r}}$ Dipartimento di Fisica dell'Università di Roma Tre and INFN Sezione Roma Tre, 00146 Roma, Italy
}

Received 1 February 2000; received in revised form 21 February 2000; accepted 22 February 2000

Editor: L. Montanet

\begin{abstract}
We present the measurement of two event samples induced by atmospheric $\nu_{\mu}$ of average energy $\bar{E}_{\nu} \sim 4 \mathrm{GeV}$. In the first sample, a neutrino interacts inside the MACRO detector producing an upward-going muon leaving the apparatus. The ratio of the number of observed to expected events is $0.57 \pm 0.05_{\text {stat }} \pm 0.06_{\text {syst }} \pm 0.14_{\text {theor }}$ with an angular distribution similar to that expected from the Bartol atmospheric neutrino flux. The second is a mixed sample of internally produced downwardgoing muons and externally produced upward-going muons stopping inside the detector. These two subsamples are selected by topological criteria; the lack of timing information makes it impossible to distinguish stopping from downgoing muons. The ratio of the number of observed to expected events is $0.71 \pm 0.05_{\text {stat }} \pm 0.07_{\text {syst }} \pm 0.18_{\text {theor }}$. The observed deficits in each subsample is in agreement with neutrino oscillations, although the significance is reduced by the large theoretical errors. However, the ratio of the two samples causes a large cancellation of theoretical and of some systematic errors. With the ratio, we rule out the no-oscillation hypothesis at $95 \%$ c.l. Furthermore, the ratio tests the pathlength dependence of possible oscillations. The data of both samples and their ratio favor maximal mixing and $\Delta m^{2} \sim 10^{-3}-10^{-2} \mathrm{eV}^{2}$. These parameters are in agreement with our results from upward throughgoing muons, induced by $\nu_{\mu}$ of much higher energies. (C) 2000 Elsevier Science B.V. All rights reserved.
\end{abstract}

The results from several underground detectors which measure the flux of atmospheric neutrinos

\footnotetext{
${ }^{1}$ Also Università della Basilicata, 85100 Potenza, Italy.

${ }^{2}$ Also INFN Milano, 20133 Milano, Italy.

${ }^{3}$ Also Istituto TESRE/CNR, 40129 Bologna, Italy.

${ }^{4}$ Also Dept. of Phys., Pennsylvania St. Univ., Univ. Park, PA 16801, USA.

${ }^{5}$ Also Università di Trieste and INFN, 34100 Trieste, Italy.

${ }^{6}$ Also Dip. di Energetica, Università di Roma, 00185 Roma, Italy.

${ }^{7}$ Also Dip. Fisica Univ. della Calabria, Rende (Cs), Italy.

${ }^{8}$ The Colorado Coll., Colorado Springs, CO 80903, USA .

${ }^{9}$ Also INR, Russian Academy of Science, 117312 Moscow, Russia.

${ }^{10}$ Also Dept. of Phys. J. Madison Univ., Harrisonburg, VA 22807, USA.

${ }^{11}$ Also Scuola Normale Superiore di Pisa, 56010 Pisa, Italy.

${ }^{12}$ Also Inst. Space Sciences, 76900 Bucharest, Romania.

${ }^{13}$ Corresponding author. E-mail: SPURIO@BO.INFN.IT.
}

give strong indication that $\nu_{\mu}$ 's oscillate into neutrinos of another type [1-3]. Fully-contained and partially-contained neutrino-induced events observed in underground detectors come from neutrinos of energy $\sim 1 \mathrm{GeV}$. The flux of atmospheric neutrinos of several tens of $\mathrm{GeV}$ can be inferred from the measurement of neutrino-induced upward-going muons that traverse the entire detector (up-throughgoing muons). The hypothesis of neutrino oscillations, with best-fit parameters $\sin ^{2} 2 \theta_{\text {mix }} \sim 1$ and $\Delta m^{2}$ in the range of a few times $10^{-3} \mathrm{eV}^{2}$, can explain the observed anomalies both in the ratio of contained $\nu_{\mu}$ to $\nu_{e}$ events (Super-K, Soudan 2) and in the zenith angle distribution of up-throughgoing muons (MACRO, Super-K).

The MACRO detector measures atmospheric neutrinos of high energy (median energy $\sim 50 \mathrm{GeV}$ ) $[3,4]$ and few $\mathrm{GeV}$ energy. The interpretation of our high energy data in terms of $\nu_{\mu}$ oscillations came 
from a deficit and from an anomalous zenith angle distribution of the observed up-throughgoing muons originating from $\nu_{\mu}$ interactions in the rock below the detector. In an updated analysis [5] with 607 observed events ( 824 expected) both the event reduction and the shape of the zenith angle distribution confirm our previous data and are in agreement with the hypothesis of $\nu_{\mu}$ oscillations. Assuming a two flavour oscillation:

$$
P\left(\nu_{\mu} \rightarrow \nu_{\mu}\right)=1-\sin ^{2} 2 \theta_{\text {mix }} \cdot \sin ^{2}\left(1.27 \cdot \Delta m^{2} L / E_{\nu}\right)
$$

we obtained maximum mixing and $\Delta m^{2} \sim 2.5 \times$ $10^{-3} \mathrm{eV}^{2} . E_{\nu}$ is the neutrino energy (obtained from Monte Carlo) and $L=2 R_{\text {Earth }} \cdot \cos \Theta$ is the neutrino path length.

Here we report on the measurement of the flux of lower energy $\left(\bar{E}_{\nu} \sim 4 \mathrm{GeV}\right)$ atmospheric neutrinos through the detection of $\nu_{\mu}$ interactions inside the apparatus (yielding partially contained upgoing and downgoing muons) and through the detection of externally produced upward-going muons stopping inside the detector [6]. For these samples, if muon neutrinos oscillate with the parameters quoted above, a constant $\sim 50 \%$ deficit in the zenith distribution is expected for events induced by upward going $\nu_{\mu}$, because of the lower energy of the parent neutrinos. Almost no reduction is expected for downward going $\nu_{\mu}$ events. Our lower energy data presented in this letter complement the higher energy data, and the results of those analyses confirm and reinforce the conclusions reached in [3-5] in terms of neutrino oscillations.

MACRO [7] is a large area, modular tracking detector located in Hall B of the Gran Sasso Underground Laboratory in Italy, with an average rock overburden of $3700 \mathrm{hg} / \mathrm{cm}^{2}$. It is a rectangular box, $76.6 \mathrm{~m} \times 12 \mathrm{~m} \times 9.3 \mathrm{~m}$, divided longitudinally into six supermodules and vertically into a lower part (4.8 $\mathrm{m}$ high) and an upper part (4.5 $\mathrm{m}$ high). The active detection elements are planes of streamer tubes for tracking and liquid scintillation counters for fast timing. The lower half of the detector is filled with streamer tube planes alternating with trays of crushed rock, which provide most of the 5.3 kton target mass for partially-contained neutrino interactions. The upper part is hollow and contains the electronics racks and work areas. There are 10 horizontal streamer tube planes in the bottom half of the detector, and 4 planes on the top, all with wire and $27^{\circ}$ stereo strip readouts. Six vertical planes of streamer tubes cover each side of the detector. The intrinsic angular resolution for muons is between $0.1^{\circ}$ and $1.0^{\circ}$ depending on the track length. The scintillator system consists of three widely-separated layers of horizontal boxes, and on each vertical side of the detector a layer of vertical boxes inserted between the streamer tubes. The time (position) resolution for muons in a scintillator box is about $500 \mathrm{ps}(\sim 11 \mathrm{~cm})$. The direction of the muons passing across MACRO is determined by the time-of-flight between two layers of scintillation counters.

The results presented in this letter come from 4.1 live years of data taking with the full detector, from April 1994 to February 1999. About $33 \times 10^{6}$ downgoing muons were collected, and were used to monitor the detector efficiency, the running conditions and the acceptance. The trigger rate due to downgoing muons is $0.3 \mathrm{~Hz}$. The trigger efficiency for each scintillation counter and for the streamer tubes was monitored over the data taking period using the downgoing muons.

Two samples of atmospheric muon neutrinos in the few-GeV energy range are measured. In the first sample (up partially-contained or IU $=$ Internal Upgoing $\mu$ events) there are (mainly) events induced by charged current (CC) interactions of upgoing $\nu_{\mu}$ inside the lower part of MACRO. An upgoing muon is produced, which crosses two scintillation layers (Fig. 1), so that the measurement of the direction is made through time-of-flight.

The second sample is a mix of upgoing and downgoing events. The partially contained downgoing events (down partially-contained or ID = Internal Downgoing $\mu$ ) are induced by downgoing $\nu_{\mu}$, interacting in the lower part of MACRO. The upgoing stopping events (UGS = Upward Going Stopping muons) are induced by interactions of upgoing $\nu_{\mu}$ below the detector yielding an upgoing muon which stops inside the detector. Both the down partially-contained and the upgoing stop events cross only the bottom layer of liquid scintillation counters (see Fig. 1) and are identified by means of topologi- 


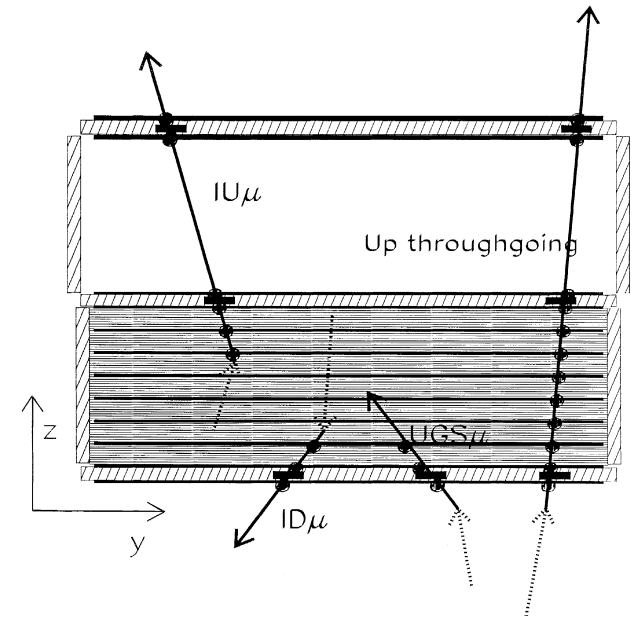

Fig. 1. Event topologies induced by neutrino interactions in or around MACRO. IU $=$ Internal Upgoing $\mu$; ID $=$ Internal Downgoing $\mu$; UGS $=$ Upgoing Stopping $\mu$; Up throughgoing $=$ upward throughgoing $\mu$. The black circles indicate the streamer tube hits, and the black boxes the scintillator hits. The TOF of the muon is measured for the IU and Up throughgoing events.

cal criteria. The lack of timing information makes it impossible to distinguish between the two subsamples. Fig. 2 shows the parent neutrino energy distribution from a Monte Carlo calculation for the three event topologies detectable in MACRO. The energy spectrum and the median energy of the two samples presented in this letter are almost the same.

The identification of IU events is based on topological criteria and time-of-flight measurements. The main requirement is the presence of at least two hit scintillator clusters, respectively in the center layer and in the upper part of the apparatus (see Fig. 1). This is the expected topology for a neutrino interacting in the lower detector and producing an upwardgoing muon with enough energy to exit the apparatus. It is also the topology of the much more numerous downgoing muons stopping in the lower detector. Scintillation timing allows the separation of the two classes of events. Moreover, the scintillators are required to match a streamer tube track reconstructed in space by our standard track-finding algorithms [7]. For IU candidates, the lowest point of a track (the starting point) must be inside the apparatus as a condition for the containment of the $\nu_{\mu}$ interaction vertex. To reject fake partially-contained events entering from an insensitive zone of the detector, the extrapolation of the track in the lower part of the detector must geometrically cross and not fire at least one scintillator layer and one streamer tube plane, or at least three planes of streamer tubes. These conditions were tuned on Monte Carlo simulated events, including evaluation of detector inefficiencies. Other cuts are applied to reject background events from downgoing atmospheric muons. They are related to the goodness of the geometrical agreement between scintillator hits and the streamer track, to the proper operation of the scintillation counters and to the quality of the time measurement. The measured $1 / \beta$ distribution after all analysis cuts (including the requirements of vertex containment) is shown in Fig. 3. The measured muon velocity $\beta c$ is evaluated with the sign convention that upgoing muons have $1 / \beta \sim-1$. A total of 121 events survive in the range $-1.3<1 / \beta<-0.7$, which is taken as the range of IU signal.

From the time distribution of Fig. 3 one expects some background events; they are mostly due to wrong time measurements or secondary particle hits, yielding an almost flat $1 / \beta$ distribution. The fit of the distribution in the range $-6.0<1 / \beta<-0.3$ to a gaussian plus a straight line gives an estimated

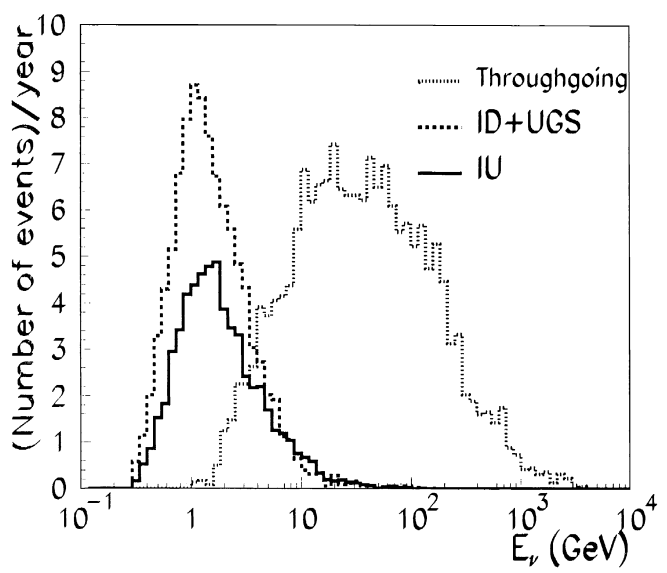

Fig. 2. Monte Carlo simulated distribution of the parent neutrino energy giving rise to the three different topologies of events detectable by MACRO. The distributions are normalized to one year of data taking; the analysis cuts are included. 


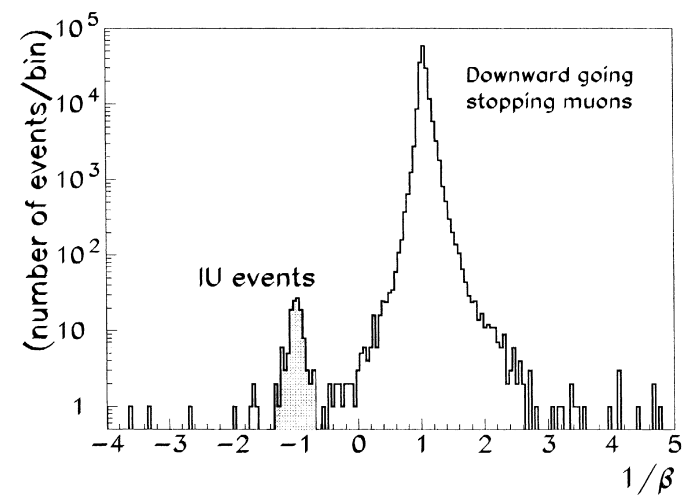

Fig. 3. The $1 / \beta$ distribution of partially-contained events after all software cuts. 121 events are in the IU event signal region, $-1.3<1 / \beta<-0.7$, with 5 estimated background events.

background of 5 events in the signal region. After background subtraction, we have 116 up partiallycontained events.

The identification of ID + UGS events is based on topological criteria. The candidates have a track starting (ending) in the lower apparatus, and crossing the bottom detector face. The track must also be located or oriented in such a way that it could not have entered or exited undetected through insensitive zones in the apparatus. Events with scintillator hits outside the bottom layer, or with the reconstructed track pointing to an insensitive zone between modules of the detector, are rejected. The standard muon tracking procedure [7] is based on at least four aligned hits in the streamer tubes. This corresponds to a minimum traversed detector thickness $t_{v} \sim 200$ $\mathrm{g} / \mathrm{cm}^{2}$ (standard sample). A relaxed retracking procedure was applied to all events with no standard track. The retracking procedure requires at least three streamer tube hits $\left(t_{v} \sim 100 \mathrm{~g} / \mathrm{cm}^{2}\right)$, aligned with respect to a fired scintillation counter in the bottom layer. The number of events with only a relaxed track is $\sim 5 \times 10^{5}$ (to be compared with the $\sim 32$ $\times 10^{6}$ standard muon tracks); these events follow the same analysis as the standard sample.

After the software cuts, 879 events survive. Some of them are tracked incorrectly (mostly by the retracking), or are bending downgoing muons, entering from an insensitive zone of the detector. Due to the bending, only a fraction of the streamer tube hits are used by the tracking algorithms. In order to reject these fake candidates, we made a double scan with the MACRO Event Display [7]. To eliminate any bias from the scan procedure, and to evaluate the absolute and relative scanning efficiencies, Monte Carlo (MC) simulated events (described below) were randomly injected into the data sample before the scanning stage. Two physicists independently scanned the merged sample. At the end of the scan, 200 events in the real data (106 ID + UGS candidates are relaxed tracked events) are accepted as upgoing stopping or partially contained downgoing muons. $97 \%$ of the real events selected by one physicist were also selected by the other.

Downgoing muons which pass near or through the detector may produce low-energy, upgoing particles, which could simulate neutrino-induced upgoing muons if the downgoing muon misses the detector. This background has been evaluated using a full simulation, based on our measurements [8]. The estimated background is $7 \pm 2$ events. A second background source could arise from atmospheric muons and detector inefficiency. Using a simulated sample of $10^{7}$ atmospheric muons, which includes measured detector inefficiencies, no events were selected by the above described procedure. After background subtraction, 193 events represent the down partially-contained plus upgoing stopping signal.

The expected number of neutrino-induced events was estimated from GMACRO [7], a GEANT-based [9] full MC detector simulation. The $\nu_{e}$ and $\nu_{\mu}$ interaction rates have been computed using the atmospheric neutrino flux of the Bartol group [10] and the neutrino cross sections of Ref. [11]. In this cross-section model, the contributions of the exclusive channels of lowest multiplicity (quasi-elastic and single pion production) are calculated separately from deep inelastic scattering (DIS). The DIS contribution to the $\nu \mathrm{N}$ cross section was computed using the GRVLO-94 [12] parton distribution functions. Using these neutrino fluxes and cross sections for $E_{\nu} \geq 300 \mathrm{MeV}$, we expect a total $(\mathrm{CC}+\mathrm{NC})$ interaction rate of $72 / \mathrm{kton} \cdot y\left(\nu_{e}+\bar{\nu}_{e}\right)$ and $150 / \mathrm{kton} \cdot y\left(\nu_{\mu}+\bar{\nu}_{\mu}\right)$. Two simulated samples have been generated, because of the different vertex locations for the IU and the ID + UGS events; the simulated events were processed with the same analysis chain as the data.

For the IU events, a sample of $10^{5}$ interactions inside the apparatus was generated (equivalent to 86 
Table 1

Summary of the properties of simulated IU and ID + UGS events. From column 2 to 5: expected event rate (no neutrino oscillations); percentages of events induced by $\nu_{\mu} \mathrm{CC}$ interactions; median parent neutrino energies (5\% of events with the highest/lowest energies were excluded); percentages of events induced by upgoing neutrinos

\begin{tabular}{lllll}
\hline & $\begin{array}{l}\text { Rate } \\
(\mathrm{ev} / \mathrm{yr})\end{array}$ & $\begin{array}{l}\nu_{\mu} \mathrm{CC} \\
(\%)\end{array}$ & $\begin{array}{l}\bar{E}_{\nu} \\
(\mathrm{GeV})\end{array}$ & $\begin{array}{l}\nu \uparrow \\
(\%)\end{array}$ \\
\hline IU & 49.3 & 87 & 4.2 & 94 \\
$\mathrm{ID}+$ UGS & 66.7 & 87 & 3.5 & 51 \\
\hline
\end{tabular}

years live time). The simulation indicates (see Table 1) that $87 \%$ of detected IU events come from $\nu_{\mu}$ charged current (CC) interactions, 9\% from $\nu_{e} \mathrm{CC}$ and the remaining fraction from neutral current (NC) interactions. Due to detector inefficiencies and analysis algorithm failures, some neutrino-induced events originating in the rock surrounding the detector are expected to contribute to the selected sample of up partially-contained events (upward-throughgoing $\mu$ 's appearing as partially-contained). The vertex containment requirements reduce this background to about $1 \%$, evaluated using a simulated sample of up throughgoing muons [3]. The fully-automated selection gives a total number of 202 expected events in the IU event signal region, $-1.3<1 / \beta<-0.7$ for $4.1 \mathrm{yr}$ of life-time.

For the ID + UGS events, $1.16 \times 10^{6}$ neutrino interactions were simulated in a larger volume (including the experimental hall, the detector and the surrounding rock). The generated events correspond to a live time of $31.1 \mathrm{yr}$. The dimensions of the interaction volume (with $13 \mathrm{~m}$ of rock below the detector, and a total rock mass of $165 \mathrm{kton}$ plus 5.3 kton of the apparatus itself) were chosen to reduce to less than $1 \%$ the number of $\nu_{\mu}$-induced stopping muons produced outside that volume. The 2199 events which survived the software selection for the ID + UGS were merged with the real events which passed the same software selection, and visually scanned. After the scan procedure 2074 (= $94.3 \%$ ) of the simulated and reconstructed events were accepted, together with the 200 real events. The expected rate is 273 events in $4.1 \mathrm{yr}$ life-time. In Table 1 we give the main features of the IU and
ID + UGS simulated samples (expected rate, percentage of $\mathrm{CC} \nu_{\mu}$ interactions, median parent neutrino energy, and fraction of events induced by upgoing neutrinos).

The number of detected IU events in the $4.1 \mathrm{yr}$ life-time is 116 , while the expected number is 202 . The ratio of the measured to the expected events is $R_{\mathrm{IU}}=\left(\frac{\text { Data }}{\mathrm{MC}}\right)_{\mathrm{IU}}=0.57 \pm 0.05_{\text {stat }} \pm 0.06_{\text {syst }} \pm 0.14_{\text {theor }}$. For the ID + UGS sample, 193 events are detected while 273 are expected. The ratio is $R_{\mathrm{ID}+\mathrm{UGS}}=0.71$ $\pm 0.05_{\text {stat }} \pm 0.07_{\text {syst }} \pm 0.18_{\text {theor }}$. Each data set is (within errors) consistent with a constant deficit (43\% for the IU sample, $29 \%$ for the ID + UGS) in all zenith bins compared to the Monte Carlo expectations assuming no oscillations. Fig. 4 shows the zenith angle distributions for the two measured data sets and for the Monte Carlo simulations. The expectations are affected by a systematic theoretical error due to the uncertainties regarding the atmospheric neutrino flux and the neutrino cross sections. At present there is no unique and reliable estimate of
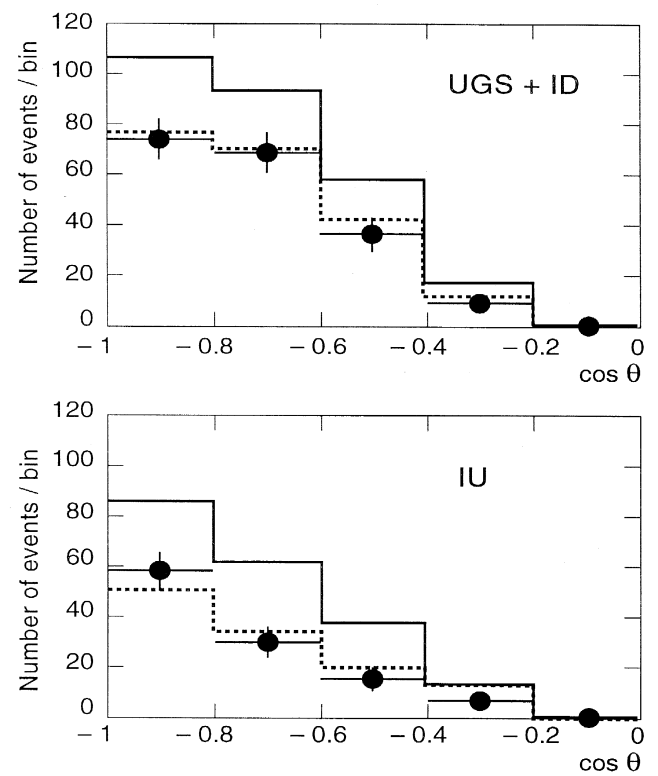

Fig. 4. Measured distributions in the cosine of the zenith angle $\Theta$ for the (a) ID + UGS and (b) IU events (black points with error bars). The solid lines are the Monte Carlo predictions assuming no oscillations. The dashed lines are the expectations for $\nu_{\mu} \rightarrow \nu_{\tau}$ oscillations with $\Delta m^{2}=2.5 \times 10^{-3} \mathrm{eV}^{2}$ and maximal mixing. 
the total theoretical uncertainty for the rate calculations. Each experimental group, and for each event category, has its own way to estimate it. For this analysis we conservatively estimate $20 \%$ for the flux and $15 \%$ for the cross section, which add in quadrature to an error of $25 \%$. For our high energy events [3] we quoted $17 \%$, while in the recent SuperKamiokande [13] analysis of neutrino-induced stopping muons $22 \%$ was quoted.

Our measured value of $R_{\mathrm{IU}}=0.57$ is quite far from the expected value of unity. If we ignore theoretical errors, an experiment with our statistical and experimental uncertainties would only fluctuate so far from unity with a probability of $2.5 \times 10^{-4}$. If we take the $25 \%$ theoretical error into account, the probability becomes $6.5 \%$. $R_{\mathrm{ID}+\mathrm{UGS}}=0.71$ also differs from unity, though not as significantly as $R_{\mathrm{IU}}$. If the observed deficits were due only to an overall theoretical overestimate [14] of the neutrino fluxes or cross sections, one would expect $R_{\mathrm{IU}} \sim R_{\mathrm{ID}+\mathrm{UGS}}$.

The theoretical uncertainties largely cancel if the ratio $\left(\frac{\mathrm{IU}}{\mathrm{ID}+\mathrm{UGS}}\right)_{\mathrm{Data}}$ is compared with the expectation $\left(\frac{\mathrm{IU}}{\mathrm{ID}+\mathrm{UGS}}\right)_{\mathrm{MC}}$. The partial error cancellation arises from the almost identical energy spectra of parent neutrinos for the two samples of events; we evaluated the remaining theoretical error as 5\%, mostly because of small differences due to residual geomagnetic effects. The experimental systematic uncertainty for the ratio is estimated at $6 \%$. The measured ratio is $\left(\frac{\mathrm{IU}}{\mathrm{ID}+\mathrm{UGS}}\right)_{\mathrm{Data}}=0.60 \pm 0.07_{\text {stat }}$. The MC predicted ratio without oscillations is $\left(\frac{\mathrm{IU}}{\mathrm{ID}+\mathrm{UGS}}\right)_{\mathrm{MC}}=$ $0.74 \pm 0.04_{\text {syst }} \pm 0.04_{\text {theor }}$. The probability to obtain a measured ratio so far from the expected MC one is $5 \%$, almost independent of the neutrino fluxes and neutrino cross sections used for the predictions.

We investigated if the observed discrepancies between data and expectations could be explained by possible systematic effects. The detector mass is known to $\pm 5 \%$. The uncertainty for the detector acceptance was estimated by comparing the shape of the zenith distribution of downward-going muons stopping inside the detector with a MC expectation based on the known rock overburden: the two distributions agree within $6 \%$. Other uncertainties arise from the life-time estimate (3\%), the effective containment of the interaction vertex depending on the simulation of the detector response to internal neutrino interactions $(4 \%)$ and the background subtrac- tion (4\%). Adding all these contributions in quadrature yields our quoted experimental systematic uncertainty of $10 \%$, too small to account for the observed discrepancy.

The number of expected events was also evaluated using the NEUGEN neutrino event generator [15] (developed by the Soudan and MINOS collaborations) as input to our MC simulations. The NEUGEN generator predicts $\sim 6 \%(5 \%)$ fewer IU (ID + UGS) events than our default generator [11], well within the estimated systematic uncertainty for neutrino cross sections $(\sim 15 \%)$.

The event distributions as a function of the muon pathlength inside the detector have also been studied, as an independent consistency check. In Fig. 5 the data (black points) are compared with the MC expectation (solid lines for no neutrino oscillations; dashed lines for oscillations at our test point).

Our data disfavor the no-oscillations hypothesis regardless of overall normalization; they are consistent with neutrino oscillations $\left(\nu_{\mu}\right.$ disappearance $)$
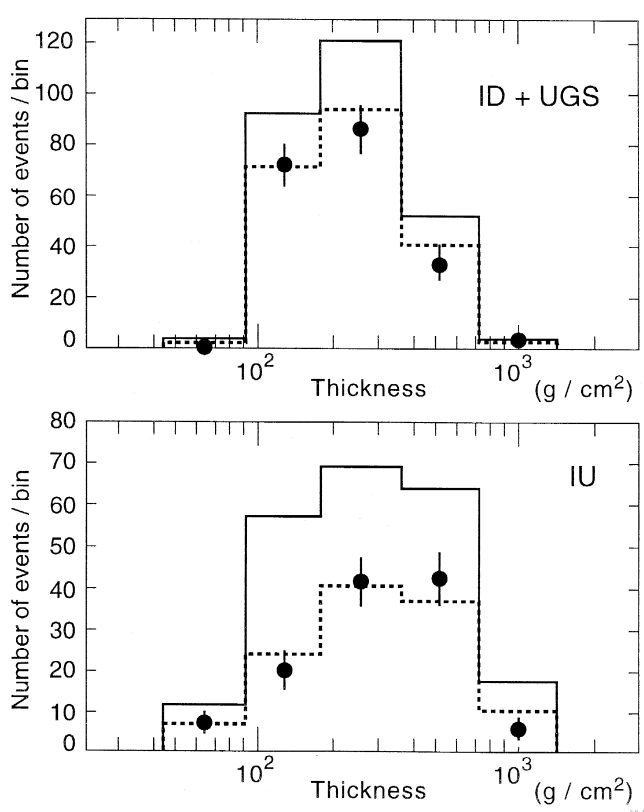

Fig. 5. Distribution of the amount of material $\left(t\right.$ in $\left.\mathrm{g} \mathrm{cm}^{-2}\right)$ in the detector traversed by IU and ID + UGS events. The data points (black points with error bars) are compared with the Monte Carlo prediction and no oscillations (solid histogram). The expectation for $\nu_{\mu} \rightarrow \nu_{\tau}$ oscillations with maximal mixing and $\Delta m^{2}=2.5 \times$ $10^{-3} \mathrm{eV}^{2}$ is shown by the dashed histogram. 
with maximal mixing and $\Delta m^{2} \sim(1-20) \times 10^{-3}$ $\mathrm{eV}^{2}$. Using the best-point parameters from our highenergy analyses [3,5], $\Delta m^{2}=2.5 \times 10^{-3} \mathrm{eV}^{2}$ and $\sin ^{2} 2 \theta_{\text {mix }}=1$, the predicted numbers of events and the angular distributions (indicated by the dashed histograms in Fig. 4 and Fig. 5) are in good agreement with the measured data.

For $\Delta m^{2} \sim(1-20) \times 10^{-3} \mathrm{eV}^{2}$, upgoing neutrinos (which induce IU and UGS events), which travel thousands of kilometers through the Earth, are reduced by $50 \%$. Almost no reduction is expected for downgoing partially-contained muons. In this scenario, and for a pure $\nu_{\mu} \mathrm{CC}$ interaction sample, the expected event rate is $1 / 2$ of the IU and $3 / 4$ of the ID + UGS predictions without oscillations. The predicted reduction for upgoing $\nu_{\mu}$ is less than $1 / 2$ because of the $\nu_{e}$ and NC event contaminations. The Monte Carlo expectation for $\nu_{\mu}$ oscillations is 115 up partially-contained events, while 202 are expected in absence of oscillations. For the up stopping plus down partially-contained events, the expectation is 202 for $\nu_{\mu}$ oscillations, while 273 events are expected for no-oscillations.

Our data disfavor $\Delta m^{2}>10^{-2} \mathrm{eV}^{2}$, for which the ID events are also reduced; both the ID + UGS and IU event rates are $\sim 1 / 2$ of the no-oscillations expectation. We also disfavor $\Delta m^{2}<10^{-3} \mathrm{eV}^{2}$, for which the shape of the angular distributions (Fig. 4) would be modified.

We estimated the most likely values of $\Delta m^{2}$ and $\sin ^{2} 2 \theta_{\text {mix }}$ using a $\chi^{2}$ method for data and Monte Carlo for the data of Fig. 4. The $\chi^{2}$ was computed with ten degrees of freedom: the histograms $(2 \times 4$ bins, normalized so as to contain only distribution shape information; the most horizontal bins were not used), the $\frac{\mathrm{IU}}{\mathrm{ID}+\mathrm{UGS}}$ ratio and the overall normalization. The statistical and systematic errors are added in quadrature; the systematic uncertainty is $10 \%$ in each bin of the angular distributions, $5 \%$ for the ratio, and $25 \%$ for the normalization. Fig. 6 shows the $90 \%$ confidence level region, based on the application of the MC prescriptions of Ref. [16] on a $\left(\sin ^{2} 2 \theta_{\text {mix }}, \Delta m^{2}\right)$ grid. The expected flux $\Phi_{\text {osc }}^{i}$ for a given point of $\left(\sin ^{2} 2 \theta_{\text {mix }}, \Delta m^{2}\right)$ in the grid is obtained by weighting each simulated event with its surviving probability $P\left(\nu_{\mu} \rightarrow \nu_{\mu}\right)$ in that bin. The maximum of the $\chi^{2}$ probability $(97 \%)$ occurs at $\sin ^{2} 2 \theta_{\text {mix }}=1.0$; this $\chi^{2}$ probability is almost con-

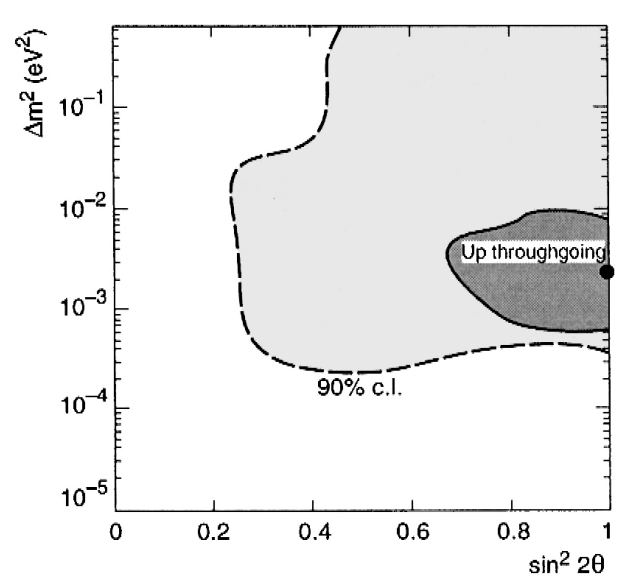

Fig. 6. Allowed contours at $90 \%$ C.L. for $\nu_{\mu} \rightarrow \nu_{\tau}$ oscillations obtained by combining the low energy neutrino events (IU and ID + UGS) using the prescription of [16]. The 90\% C.L. contour and the best fit point from our high-energy analysis [5] is also shown as a solid line.

stant in the interval $\Delta m^{2}=(1-20) \times 10^{-3} \mathrm{eV}^{2}$. In the region of the maximum, the flux normalization factor is 1.02 in both data sets (i.e. the data are $2 \%$ higher than the oscillated predictions with our normalization).

In conclusion, we presented measurements of two samples of events induced by relatively low-energy neutrinos $\left(\bar{E}_{\nu} \sim 4 \mathrm{GeV}\right)$ interacting in MACRO or in the surrounding rock. The neutrinos originate from cosmic ray interactions in the upper atmosphere above the detector (downgoing events) or up to $\sim 13000 \mathrm{~km}$ away on the opposite side of the Earth (upgoing events). The ratio of the number of observed to expected events (no oscillations) is $0.57 \pm$ $0.05_{\text {stat }} \pm 0.06_{\text {syst }} \pm 0.14_{\text {theor }}$ for the IU sample and $0.71 \pm 0.05_{\text {stat }} \pm 0.07_{\text {syst }} \pm 0.18_{\text {theor }}$ for the ID + UGS sample. Within statistics, the observed deficits are uniform over the zenith angle. The ratio of IU to ID + UGS disfavors the hypothesis that the reduction is due to a systematic uncertainty in the event prediction rate. The hypothesis of muon neutrino oscillations explains the different deficits in IU and ID + UGS events with higher probability. The large theoretical uncertainties for the neutrino flux and cross sections is dominant in each data set; the ratio of the two low energy samples is dominated by statistical uncertainties. The regions with $\Delta m^{2}>3$ $\times 10^{-4} \mathrm{eV}^{2}$ and $\sin ^{2} 2 \theta_{\text {mix }}>0.25$ are allowed at 
90\% C.L. The best region corresponds to maximal mixing and $\Delta m^{2}=(1-20) \times 10^{-3} \mathrm{eV}^{2}$. This result confirms the scenario proposed by the measurement of higher-energy neutrino-induced muons by MACRO [3,5], as well as by other experiments [1,2], all of which favor the $\nu_{\mu}$ oscillation hypothesis with maximal mixing and $\Delta m^{2}$ of a few times $10^{-3} \mathrm{eV}^{2}$.

\section{Acknowledgements}

We acknowledge the support of the staff of the Gran Sasso Laboratory and of the Institutions participating in the experiment. We thank the Istituto Nazionale di Fisica Nucleare (INFN), the US Department of Energy and the US National Science Foundation for their support. We thank INFN, FAI, ICTP (Trieste), NATO and WorldLab for providing fellowships and grants for non-Italian citizens.

\section{References}

[1] Super-Kamiokande Collaboration, Y. Fukuda et al., Phys. Rev. Lett. 81 (1998) 1562.
[2] Soudan 2 Collaboration, W.W.M. Allison et al., Phys. Lett. B 449 (1999) 137.

[3] MACRO Collaboration, M. Ambrosio et al., Phys. Lett. B 434 (1998) 451.

[4] MACRO Collaboration, M. Ambrosio et al., Phys. Lett. B 357 (1995) 481.

[5] MACRO Collaboration, Neutrino oscillations at high energies by MACRO, INFN/AE-99/09 hep-ex/9905025.

[6] Preliminary results were presented at the conferences/workshops of: Alcalà de Henares 1998, hep-ex/9808001; Vulcano 1998, hep-ex/9809003; Takayama 1998, hepex/9810008; DPF 1999, hep-ex/9903030; La Thuile 1999, hep-ex/9906019; ICRC 1999, INFN/AE-99/10, 1999.

[7] MACRO Collaboration, S. Ahlen et al., Nucl. Instr. and Meth. A 324 (1993) 337.

[8] MACRO Collaboration, M. Ambrosio et al., Astropart. Phys. 9 (1998) 105.

[9] R. Brun et al., CERN report DD/EE 84-1, 1987.

[10] V. Agrawal et al., Phys. Rev. D 53 (1996) 1314.

[11] P. Lipari et al., Phys. Rev. Lett. 74 (1995) 4384.

[12] M. Glück et al., Z. Phys. C 67 (1995) 433.

[13] Super-Kamiokande Collaboration, Y. Fukuda et al., Neutrino-induced upward stopping muons in Super-Kamiokande, hep-ex/9908049-v3, 1 December 1999.

[14] G. Battistoni et al., hep-ph/9907408, to be published in Astropart. Phys.

[15] Private communication from MINOS Collaboration,; see also H.M. Gallagher, Neutrino Oscillation Searches with the Soudan 2 Detector, Ph.D. thesis, University of Minnesota, 1996.

[16] G. Feldman, R. Cousins, Phys. Rev. D 57 (1998) 3873. 